\title{
SÍNTESE DE FLUXOGRAMAS ALTERNATIVOS PARA O PROCESSO DE GERAÇÃO DE ENERGIA A PARTIR DE RSU
}

\author{
Júlia Pancini de Oliveira \\ Universidade Federal do Rio de Janeiro / Escola de Química \\ Av. Athos da Silveira Ramos, 149, Bloco E, Ilha do Fundão, Rio de Janeiro - Brasil \\ juliapancini26@gmail.com
}

\begin{abstract}
Ana Mehl
Universidade Federal do Rio de Janeiro / Escola de Química

Av. Athos da Silveira Ramos, 149, Bloco E, Sala 209, Ilha do Fundão, Rio de Janeiro -Brasil mehl@eq.ufrj.br

Fernando Luiz Pellegrini Pessoa

Universidade Federal do Rio de Janeiro / Escola de Química

Av. Athos da Silveira Ramos, 149, Bloco E, Ilha do Fundão, Rio de Janeiro - Brasil

pessoa@eq.ufrj.br
\end{abstract}

\section{RESUMO}

O presente trabalho caracteriza o cenário dos resíduos sólidos urbanos (RSU) no estado do Rio de Janeiro, e identifica as tecnologias disponíveis para a geração de energia a partir dos resíduos do RJ. Baseado na teoria de síntese de processos e abordando as tecnologias disponíveis na literatura, foi possível dividir o processo em 7 subsistemas, cada um com pelo menos duas alternativas. Assim, obteve-se 1.440 fluxogramas alternativos, representados por uma superestrutura. A partir da superestrutura, aliado ao conhecimento do cenário e das tecnologias, foram estabelecidas regras heurísticas, com as quais é possível identificar o fluxograma mais promissor. Dentro do contexto do estado, a configuração mais promissora encontrada é o aproveitamento energético por meio da tecnologia de combustão. Com coleta efetiva, e $100 \%$ do RSU do estado encaminhado para o tratamento, é possível gerar até 14.256.095 MWh por ano, correspondendo à 94\% da demanda energética da capital. A partir do fluxograma mais promissor, será possível otimizar o sistema, encontrando o fluxograma ótimo para o processo analisado.

Palavra-chave: PO na Área de Energia; Síntese de Processos; Resíduo Sólido Urbano; Bioenergia; Waste-to-energy.

\begin{abstract}
The present work characterizes the municipal solid waste (MSW) scenario in the state of Rio de Janeiro and detects the available technologies for the generation of energy from the local waste. Through the theory of process synthesis and with the available technologies, the process was divided into seven subsystems, each one presents two or more alternatives. As a result, was detected 1,440 viable flowcharts, represented by a superstructure of alternatives. From the superstructure, in combination with the scenario and
\end{abstract}


the technologies detects, heuristics rules were established. Through the heuristics rules the most promising configuration found is the energetic use with the combustion technology in which the state of Rio de Janeiro presented a high potential of energy generation. With all the waste generated in the state of Rio de Janeiro being forwarded to treatment, the state is able to generate up to 14,256,095 MWh per year, corresponding of $94 \%$ of the city demand. From the most promising configuration, it will be possible to optimize the system, finding the optimal flowchart for the analyzed process.

Keywords: Energy Operational Research; Process synthesis; Municipal solid waste; Bioenergy; Waste-to-energy.

\section{Como Citar:}

OLIVEIRA, Júlia Pancini; MEHL, Ana; PESSOA, Fernando Luiz Pellegrini. Síntese de Fluxogramas Alternativos para o Processo de Geração de Energia a partir de RSU. In: SIMPÓSIO DE PESQUISA OPERACIONAL E LOGÍSTICA DA MARINHA, 19., 2019, Rio de Janeiro, RJ. Anais [...]. Rio de Janeiro: Centro de Análises de Sistemas Navais, 2019.

\section{INTRODUÇÃO}

A gestão dos resíduos sólidos urbanos (RSUs) é um problema global. Ao projetar o valor apurado pelo Banco Mundial em 2012, com base no crescimento populacional, estimase que atualmente sejam gerados 1,4 bilhão de tonelada de RSU anualmente no mundo, e espera-se um aumento de 800 milhões de toneladas até 2025 (HOORNWEG, 2012). A partir dos valores apresentados no Relatório Síntese do Plano Estadual de Resíduos Sólidos do Rio de Janeiro em 2013, estima-se que hoje, o estado do Rio de Janeiro gere 6,7 milhões de toneladas de RSU por ano (SEA, 2013).

A atual gestão dos RSUs não é sustentável por apresentar problemas como o aumento na geração, a falta de coleta (tanto comum como seletiva) e disposição final inadequada. Esse último pode gerar impactos ambientais como contaminação de águas pelo lixiviado, poluição do ar por queima indevida, emissão de gases de efeito estufa em aterros e lixões (HOORNWEG, 2012). Uma forma de tornar esse quadro sustentável é a utilização da hierarquia de gerenciamento de resíduos, apresentada pela Política Nacional de Resíduos Sólidos, em que é proposta uma ordem de preferência para a destinação final dos RSUs. Essa ordem é: redução na geração de resíduos, reúso dos resíduos gerados, reciclagem e recuperação, compostagem e digestão da matéria orgânica, incineração com recuperação energética e, por fim, a destinação em aterro sanitário para o mínimo de resíduo remanescente (BRASIL, 2010).

Ou seja, uma das soluções é a geração de valor a partir dos RSUs, que podem ser, por exemplo, utilizados para recuperação energética. No entanto, apesar da recuperação energética ser prevista na Política Nacional de Resíduos Sólidos como uma disposição final preferencial em relação aos aterros, menos de 0,1\% dos RSUs do Brasil é utilizado para recuperação energética (BRASIL, 2010). No estado do Rio de Janeiro, 96\% dos RSUs são coletados, mas 90\% são encaminhados à aterros (SEA, 2013).

Com isso, o presente estudo tem como objetivo propor fluxogramas alternativos por meio da metodologia de síntese de processos para geração de energia a partir do aproveitamento de resíduos sólidos urbanos. Também é identificado o fluxograma base mais promissor a partir de regras heurísticas aqui propostas. Para isso, foi realizada a 
caracterização dos RSUs do estado do Rio de Janeiro bem como a identificação das tecnologias disponíveis na literatura para o processo em questão.

\section{METODOLOGIA}

A metodologia proposta para este estudo é representada na Figura 1 e detalhada a seguir.

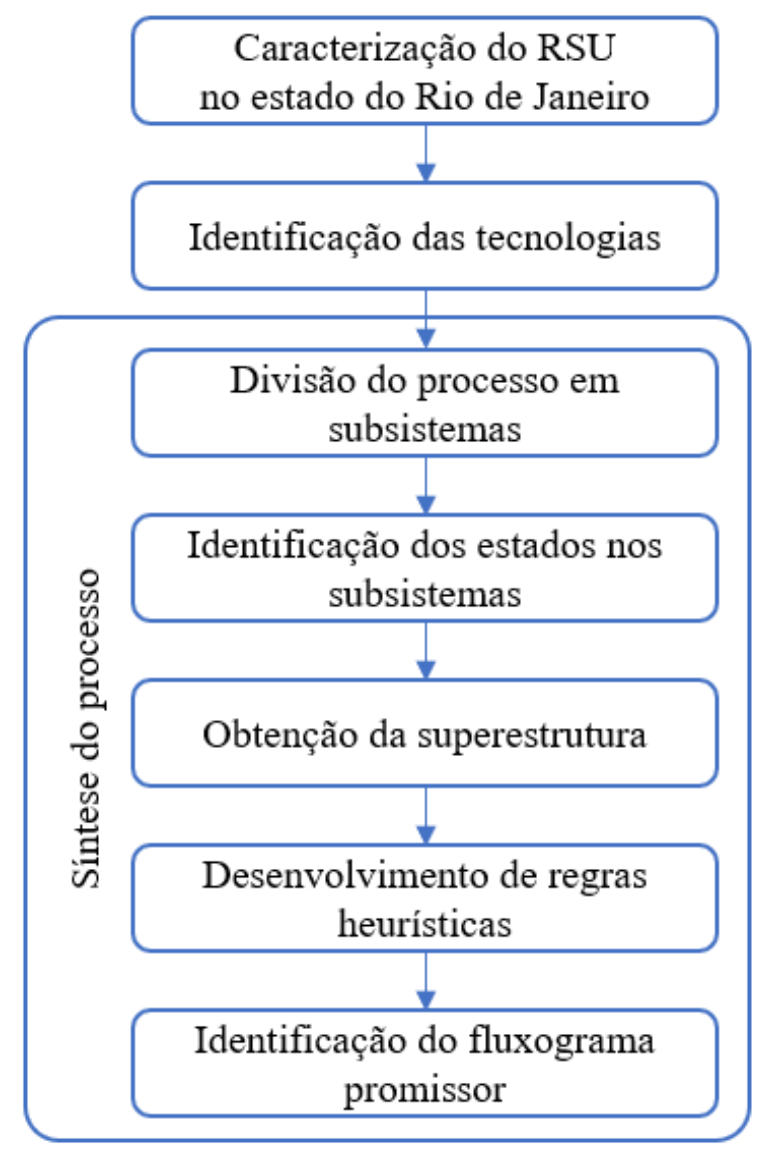

Figura 1 Fluxograma desenvolvido para a metodologia

Nesse estudo inicialmente foi realizada a caracterização dos resíduos sólidos urbanos do estado do Rio de Janeiro e em seguida a identificação das tecnologias disponíveis na literatura. Para isso foi realizada uma revisão bibliográfica em busca da análise gravimétrica dos RSU do estado e da identificação das tecnologias usadas mundialmente para o processo de geração de energia a partir de RSU. A gravimetria do RSU é importante por possibilitar a compreensão do que compõem o RSU e como esses materiais podem ser valorados na geração de energia,

Tendo identificado as tecnologias e o cenário do RSU no Rio de Janeiro, com base na teoria de síntese de processos, o processo de geração de energia a partir de RSU foi dividido em sete subsistemas: separação na fonte, triagem mecânica, tecnologia, redução granulométrica, secagem, pré-tratamento químico e conversão. Cada um desses subsistemas apresenta pelo menos dois estados como alternativas para realizar o processo, sendo que tecnologia apresenta cinco estados, redução granulométrica três e conversão seis. Com isso, obtém-se 1.440 possibilidades de fluxogramas, representados em uma superestrutura. A partir da superestrutura, aliada ao cenário do estado do Rio de Janeiro, foram estabelecidas regras heurísticas para a identificação do fluxograma mais promissor. 


\section{RESULTADOS E DISCUSSÃO}

\subsection{ResíDuOS}

Resíduo sólido é todo material em estado sólido; semi-sólido ou gasoso em recipientes; e líquido inviável de ser lançado em corpos líquidos, resultante de atividade humana que não possui mais utilidade imediata e será conduzido à destinação final (BRASIL, 2010). Os resíduos podem ser classificados quanto à origem e à periculosidade. Os RSUs são os resíduos sólidos provenientes de atividades domésticas e do serviço de limpeza urbana. Quanto à periculosidade são classificados como não perigosos não inertes, por serem combustíveis, biodegradáveis ou solúveis em água.

A Figura 2 apresenta a gravimetria em massa do RSU do estado do Rio de Janeiro (SEA, 2013). A fração orgânica é a mais representativa, com 53\% dos RSUs, seguida pelas frações recicláveis de plástico (19\%) e papel e papelão (16\%) (SEA, 2013). Esses três principais componentes representam 88\% dos RSUs do estado, e há possibilidade de gerar valor ao RSU a partir deles. A grande fração de matéria orgânica evidencia um potencial de geração de energia com o biogás.

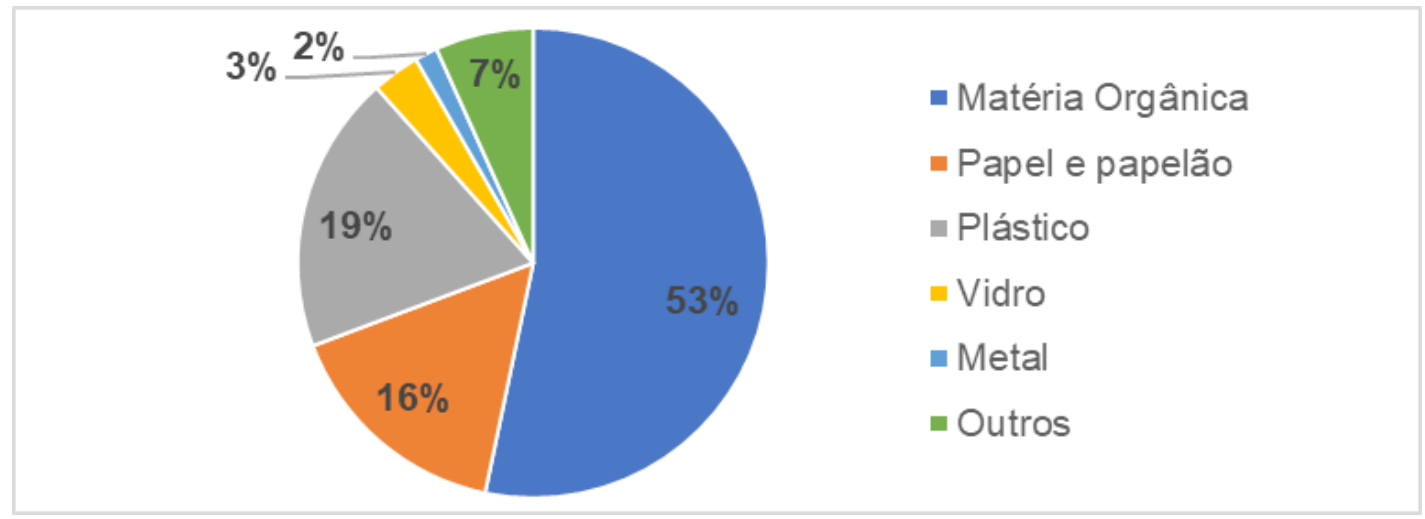

Figura 2 Composição global do RSU do estado do Rio de Janeiro

Fonte: adaptado de SEA, (2013)

Estima-se que o estado do Rio de Janeiro gere anualmente cerca de 6,7 milhões de toneladas de RSU (SEA, 2013). Ainda segundo a Secretaria do Estado do Ambiente, desse total gerado, $96 \%$ são coletados, mas apenas 61,6 mil toneladas são atendidos pela coleta seletiva, o que representa menos de 1\% do RSU total do estado (SEA, 2013).

\subsection{TECNOLOGIAS}

Para que o RSU possa ser aproveitado na produção de energia é importante o conhecimento das alternativas tecnológicas disponíveis mundialmente. A Tabela 1 apresenta um consolidado dessas tecnologias, com os requisitos necessários para o tratamento do RSU e aspectos positivos e negativos de cada alternativa.

Para a hierarquização das tecnologias, além dos aspectos apresentados na Tabela 1, um fator decisivo é a produção energética por tonelada de RSU tratado. A Tabela 2 apresenta os valores apurados para cada tecnologia. A literatura não apresenta um valor para comparação de produção energética para a hidrólise, e por essa razão essa tecnologia não está relacionada na Tabela 2. 
Tabela 1 Tabela comparativa das alternativas tecnológicas para o aproveitamento energético

\begin{tabular}{|c|c|c|c|c|c|}
\hline Tecnologia & $\begin{array}{l}\text { Pré- } \\
\text { tratamento }\end{array}$ & $\begin{array}{l}\text { Matéria- } \\
\text { prima }\end{array}$ & $\begin{array}{l}\text { Produto e } \\
\text { Produto } \\
\text { comercializável } \\
\end{array}$ & $\begin{array}{l}\text { Aspectos } \\
\text { positivos }\end{array}$ & $\begin{array}{l}\text { Aspectos } \\
\text { negativos }\end{array}$ \\
\hline $\begin{array}{l}\text { Digestão } \\
\text { Anaeróbia }\end{array}$ & $\begin{array}{l}\text { Mecânico: } \\
\text { triagem; } \\
\text { Trituração }\end{array}$ & $\begin{array}{l}\text { Matéria } \\
\text { orgânica }\end{array}$ & $\begin{array}{l}\text { Biogás } \\
\text { Energia }\end{array}$ & $\begin{array}{l}\text { Único dos } \\
\text { processos citados } \\
\text { que pode tratar } \\
\text { RSU com alta } \\
\text { umidade e baixo } \\
\text { poder calorífico } \\
\text { sem secagem; } \\
\text { Tecnologia } \\
\text { consolidada em } \\
\text { larga escala }\end{array}$ & $\begin{array}{l}\text { Tempo; } \\
\text { Trata apenas uma } \\
\text { parcela do RSU; } \\
\text { Para os processos } \\
\text { úmidos, o RSU } \\
\text { precisa ser } \\
\text { misturado à lodo } \\
\text { industrial; } \\
\text { Exige pré- } \\
\text { tratamento }\end{array}$ \\
\hline Pirólise & $\begin{array}{l}\text { Mecânico: } \\
\text { triagem; } \\
\text { Moagem; } \\
\text { Secagem }\end{array}$ & $\begin{array}{l}\text { Matéria } \\
\text { orgânica; } \\
\text { Plástico; } \\
\text { Papel }\end{array}$ & $\begin{array}{l}\text { Bio-óleo; } \\
\text { Carvão; } \\
\text { Gás de Síntese } \\
\text { Energia; } \\
\text { Produtos } \\
\text { Químicos }\end{array}$ & $\begin{array}{l}\text { Pode ser } \\
\text { utilizada no } \\
\text { tratamento de } \\
\text { mais de uma } \\
\text { parcela do RSU; } \\
\text { Produz fração } \\
\text { líquida: facilita } \\
\text { transporte e } \\
\text { estocagem }\end{array}$ & $\begin{array}{l}\text { Exige pré- } \\
\text { tratamento }\end{array}$ \\
\hline
\end{tabular}

\begin{tabular}{|c|c|c|c|c|c|}
\hline $\begin{array}{l}\text { Gaseificaçã } \\
\text { o }\end{array}$ & $\begin{array}{l}\text { Mecânico: } \\
\text { triagem; } \\
\text { Redução } \\
\text { granulomé- } \\
\text { trica }\end{array}$ & $\begin{array}{l}\text { Matéria } \\
\text { orgânica; } \\
\text { Plástico; } \\
\text { Papel }\end{array}$ & $\begin{array}{l}\text { Gás de Síntese } \\
\text { Energia; } \\
\text { Produtos } \\
\text { Químicos }\end{array}$ & $\begin{array}{l}\text { Pode ser } \\
\text { utilizada no } \\
\text { tratamento de } \\
\text { mais de uma } \\
\text { parcela do RSU }\end{array}$ & $\begin{array}{l}\text { Exige pré- } \\
\text { tratamento; } \\
\text { Alto custo de } \\
\text { operação e } \\
\text { manutenção; } \\
\text { Combustão } \\
\text { incompleta }\end{array}$ \\
\hline
\end{tabular}

\begin{tabular}{llllll} 
Combustão & $\begin{array}{l}\text { Mecânico: } \\
\text { triagem } \\
\text { (facultativo) }\end{array}$ & $\begin{array}{l}\text { Exceto } \\
\text { vidro } \\
\text { e metal }\end{array}$ & $\begin{array}{l}\text { Energia elétrica; } \\
\text { Aquecimento/ } \\
\text { Resfriamento }\end{array}$ & $\begin{array}{l}\text { Tecnologia } \\
\text { consolidada em } \\
\text { larga escala }\end{array}$ & $\begin{array}{l}\text { Risco de } \\
\text { vazamento nas } \\
\text { grades }\end{array}$ \\
Hidrólise & $\begin{array}{l}\text { Mecânico: } \\
\text { triagem; }\end{array}$ & & Glicose & Exige pré- \\
Moagem; & $\begin{array}{l}\text { Secagem; } \\
\text { Ácido ou } \\
\text { básico } \\
\text { (enzimática) }\end{array}$ & Papel & Etanol & químico & $\begin{array}{l}\text { tratamento; } \\
\text { Trata apenas uma } \\
\text { parcela do RSU; } \\
\text { Tecnologia não } \\
\text { consolidada em } \\
\text { escala industrial }\end{array}$ \\
\hline
\end{tabular}

Fonte: autoria própria com informações de ARENA (2012), EUROPEAN COMMISSION (2006a,b), KALOGIROU (2018), KHAN (1992), PEDROZA et al. (2017) e ROGOFF; SCREVE (2011) 
Tabela 2 Energia média gerada por tonelada de RSU para cada alternativa tecnológica

\begin{tabular}{lcc}
\hline Tecnologia & $\begin{array}{c}\text { Energia por } \\
\text { tonelada de RSU } \\
\text { (MWh/t) }\end{array}$ & Desvio padrão \\
\hline Digestão anaeróbia & 0,28 & 0,24 \\
Pirólise & 0,24 & 0,04 \\
Gaseificação & 1,46 & 0,79 \\
Combustão & 2,42 & 0,79 \\
\hline
\end{tabular}

Fonte: autoria própria com informações de ARENA (2012), EPA (2018), EUROPEAN COMMISSION (2006b), HOGG et al. (2002), ISWA (2012) e PEDROZA et al. (2017)

A partir dos dados apresentados nas Tabelas 1 e 2, aliados à gravimetria em massa do RSU do estado do Rio de Janeiro e à quantidade de RSU gerado no estado foi possível realizar uma estimativa do potencial energético dos resíduos sólidos urbanos do Rio de Janeiro. A massa de RSU possível de ser tratada por cada tecnologia, é determinada com o percentual - apresentado na gravimetria da Figura 2 - da fração do RSU possível de ser utilizada como matéria prima para a alternativa, considerando que $100 \%$ do resíduo do estado seja coletado e encaminhado para o tratamento. A Tabela 3 apresenta o potencial energético para cada tecnologia ressaltando o resultado de potencial de geração de 14.256.095 MWh por ano, pela tecnologia de combustão. Essa geração energética corresponde à $94 \%$ da demanda da capital do estado.

Tabela 3 Potencial energético no estado do Rio de Janeiro para cada alternativa tecnológica

\begin{tabular}{lcc}
\hline Tecnologia & $\begin{array}{c}\text { Potencial de } \\
\text { RSU tratado (t) }\end{array}$ & $\begin{array}{c}\text { Potencial energético no } \\
\text { estado do Rio de Janeiro } \\
\text { (MWh) }\end{array}$ \\
\hline Digestão anaeróbia & 3.550 .161 & 994.045 \\
Pirólise & 5.890 .949 & 1.413 .828 \\
Gaseificação & 5.890 .949 & 8.600 .785 \\
Combustão & 5.890 .949 & 14.256 .095 \\
\hline
\end{tabular}

Fonte: autoria própria com informações de ARENA (2012), EPA (2018), EUROPEAN COMMISSION (2006b), HOGG et al. (2002), ISWA (2012), PEDROZA et al. (2017) e SEA (2013)

\subsection{SÍNTESE DE FLUXOGRAMAS PARA O CASO RSU - ENERGIA}

A fim de identificar o fluxograma mais promissor pelo método heurístico, inicialmente, dividiu-se o processo em sete subsistemas: separação na fonte (1), triagem mecânica (2), tecnologia (3), redução granulométrica (4), secagem (5), pré-tratamento químico (6) e conversão (7). Cada subsistema apresenta mais de uma possibilidade, e estão descritos a seguir.

(1) A separação na fonte facilita a etapa de triagem mecânica. Para isso o país precisa disponibilizar estrutura viável para a população realizá-la. Por isso é avaliada a possibilidade com separação na fonte (SF) e sem separação na fonte (SSF);

(2) O pré-tratamento por triagem mecânica separa os resíduos que podem ser tratados pela tecnologia adotada. Essa triagem pode ser complexa (TMC) com triturador para abertura de sacos e com peneira, separação por ar e magnética para segregação de diferentes 
materiais; ou simples (TMS) para uma separação adicional, sem o triturador, nesse caso quando o RSU já vem separado na fonte;

(3) Nesse subsistema há a aplicação de uma das cinco tecnologias, sendo elas: digestão anaeróbia (TDA), pirólise (TP), gaseificação (TG), combustão (TC) e por fim, hidrólise (TH);

(4) Em alguns casos é importante pré-tratamento com homogeneização e redução de tamanho para facilitar o processamento do resíduo. Dessa forma, são avaliadas duas possibilidades de redução granulométrica, por moagem (RGM) ou utilizando triturador (RGT). É importante observar que nos casos em que são realizados a moagem é necessário passar previamente por um triturador. Além disso, também é analisada a possibilidade de não realizar a redução (SRG);

(5) Para alguns processos é necessário realizar pré-tratamento com secagem. Dessa forma, nesse nível, será analisado o caso com secagem (CS) e sem secagem (SS);

(6) Além dos pré-tratamentos físicos mencionados nos subsistemas anteriores, pode haver a necessidade de pré-tratamento químico. No sexto nível é analisada a necessidade de pré-tratamento químico (PTQ) ou não (SPTQ).

(7) O último subnível apresenta a conversão utilizada para transformar o produto obtido no processo no produto comercializável, ou seja, o produto desejado, energia. Essa conversão pode ser por meio de processos químicos ou bioquímicos (CPQ), turbina (CT), caldeira (CC), motor (CM), caldeira e turbina (CCT) ou fermentação (CF).

Com as possibilidades em cada subsistema, é possível gerar 1.440 fluxogramas, que estão representados pela superestrutura apresentada na Figura 3. Devido ao elevado número de fluxogramas, é importante uma abordagem sistemática para eliminação dos ramos menos promissores. Dessa forma, foram estabelecidas regras heurísticas para cada subnível. 


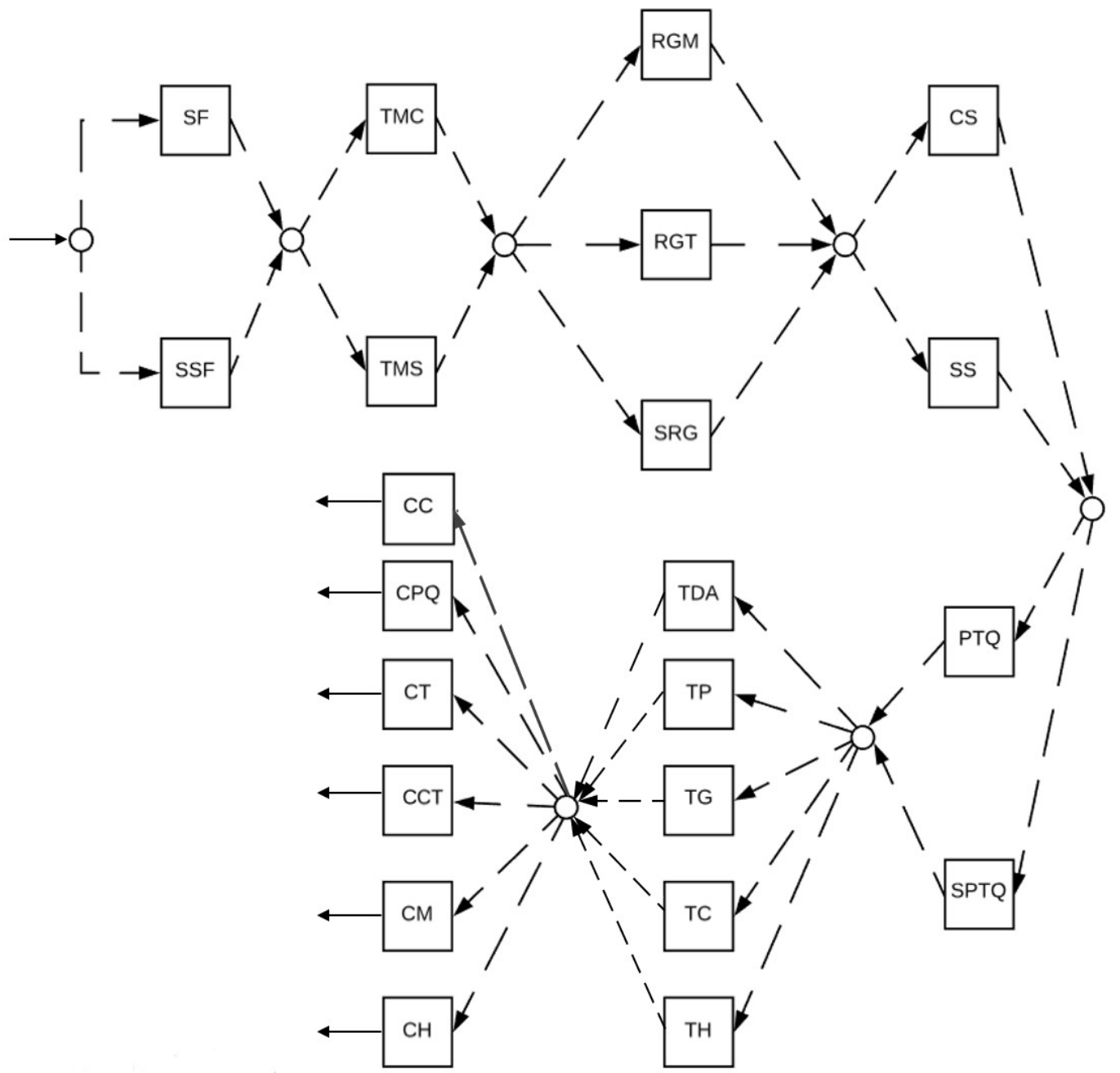

Figura 3 Representação da superestrutura para o processo de geração de energia a partir de RSU

Levando em consideração a hierarquia de gerenciamento de resíduos e a economia de escala para os custos de uma planta, é possível estabelecer duas regras para o primeiro subsistema:

Regra 1.1 - Se a coleta seletiva recolhe apenas material para reciclagem: SSF;

Regra 1.2 - Se a coleta seletiva recolher resíduos que não podem ser reciclados em um total acima de 200.000 toneladas de RSU separado na fonte por ano: SF.

Como no Rio de Janeiro, apenas cerca de 61,6 mil toneladas de RSUs recicláveis são coletadas pela coleta seletiva (SEA, 2013), para o fluxograma mais promissor o método heurístico indica a utilização do processo SSF.

A triagem mecânica é facultativa apenas para a tecnologia de combustão, no entanto, mesmo para essa tecnologia a triagem auxilia o processo, ao remover materiais com baixo poder calorífico. Com isso, é recomendável a triagem mecânica em todos os processos, e sua complexidade depende da utilização de separação na fonte. Desse modo, para o segundo subsistema, foi estabelecida uma regra heurística: TMC.

Regra 2.1 - Se o resíduo utilizado teve separação na fonte: TMS, caso contrário:

Como foi indicado o processo SSF, pela regra 2.1 estabelece-se TMC.

O terceiro subsistema define a tecnologia que deve ser utilizada, e apresenta cinco regras: 
Regra 3.1 - Se o interesse for em produção de biogás: TDA;

Regra 3.2 - Se o interesse for em produção de bio-óleo ou carvão: TP;

Regra 3.3 - Se o interesse for em produção de gás de síntese: TG;

Regra 3.4 - Se o interesse for em produção de bioetanol: TH;

Regra 3.5 - Se o interesse for em produção de energia térmica ou elétrica: TC.

As regras foram estabelecidas, de acordo com a identificação das tecnologias, apresentada na Tabela 1. Como a digestão anaeróbia é a tecnologia que produz biogás, se o interesse for a obtenção desse gás, a tecnologia aplicada deve ser a de digestão anaeróbia, como estabelecido na regra 3.1. A regra 3.2 é embasada na tecnologia de pirólise, única que gera bio-óleo e carvão como principais produtos. O principal produto da gaseificação é o gás de síntese, dessa forma a regra 3.3 estabelece o uso dessa tecnologia se o interesse for a produção desse gás. O etanol é o produto da tecnologia de hidrólise a partir de RSU, o que justifica a regra 3.4. A regra 3.5 é embasada na identificação da tecnologia de combustão e na hierarquização das tecnologias pela eficiência energética. A Tabela 2 identifica a tecnologia de combustão como a de maior potencial de geração energética por tonelada de RSU. A Tabela 3 apresenta os potenciais de geração energética no estado do Rio de Janeiro, identificando também a tecnologia de combustão como a de maior potencial.

Como o objetivo do estudo é o de produção de energia, pela regra 3.5 define-se o fluxograma mais promissor com a tecnologia de combustão, devido à maior produção de energia por tonelada de RSU, como apresentado nas Tabelas 2 e 3.

Para os subsistemas quatro, cinco e seis são estabelecidas quatro, três e uma regras, respectivamente. As oito regras estão baseadas na tecnologia definida no terceiro nível:

Regra 4.1 - Se o processo utilizar tecnologia de combustão: SRG;

Regra 4.2 - Se o processo utilizar as tecnologias de pirólise e hidrólise: RGM;

Regra 4.3 - Se o processo utilizar TG com gaseificador de fluxo de entrada: RGM;

Regra 4.4 - Se o processo utilizar a TDA ou TG com gaseificador que não seja de fluxo de entrada: RGT;

Regra 5.1 - Se o processo utilizar as TDA, TG ou TC: SS;

Regra 5.2 - Se o resíduo a ser utilizado não estiver úmido - não contém matéria orgânica - e o processo utilizar a TP: SS. Caso seja TH: CS;

Regra 5.3 - Se o resíduo a ser utilizado estiver úmido e o processo utilizar TP ou TH: CS;

Regra 6.1 - Se o processo utilizar TH enzimática: PTQ. Caso utilize outra tecnologia: SPTQ.

Como a tecnologia definida como mais promissora é a de combustão, a regra 4.1 indica que para o quarto nível é recomendado processo sem redução granulométrica (SRG). A regra 5.1 indica que não é necessário realizar secagem, logo, processo SS. E a regra 6.1 indica a não necessidade de pré-tratamento químico, SPTQ.

Para o sétimo subsistema foram estabelecidas seis regras:

Regra 7.1 - Se o interesse for em etanol: CF;

Regra 7.2 - Se o interesse for em biocombustíveis ou outros produtos químicos: CPQ;

Regra 7.3 - Se o interesse for em energia térmica: CC;

Regra 7.4 - Se o interesse for em eletricidade e foi gerado biogás: CT ou CM;

Regra 7.5 - Se o interesse for em eletricidade e foi gerado gás de síntese: CT ou CM;

Regra 7.6 - Se o interesse for em eletricidade e foi utilizada a tecnologia de combustão: CCT.

Como o interesse é produção energética e foi definida a tecnologia de combustão no terceiro subsistema, o método heurístico indica o processo de conversão por meio de caldeira 
e turbina (CCT).

Ao aplicar o método heurístico no problema de geração de energia a partir de RSU para o caso do estado do Rio de Janeiro, encontra-se um fluxograma promissor para o processo, que consiste em:

(1) Separação na fonte: sem separação na fonte (SSF);

(2) Triagem mecânica: triagem mecânica complexa (TMC);

(3) Redução granulométrica: sem redução granulométrica (SRG);

(4) Secagem: sem secagem (SS);

(5) Pré-tratamento químico: sem pré-tratamento químico (SPTQ);

(6) Tecnologia: tecnologia de combustão (TC);

(7) Conversão: conversão por meio de caldeira e turbina (CCT)

O fluxograma encontrado - SSF, TMC, SRG, SS, SPTQ, TC, CCT - está representado na Figura 4 na superestrutura, por meio de setas contínuas.

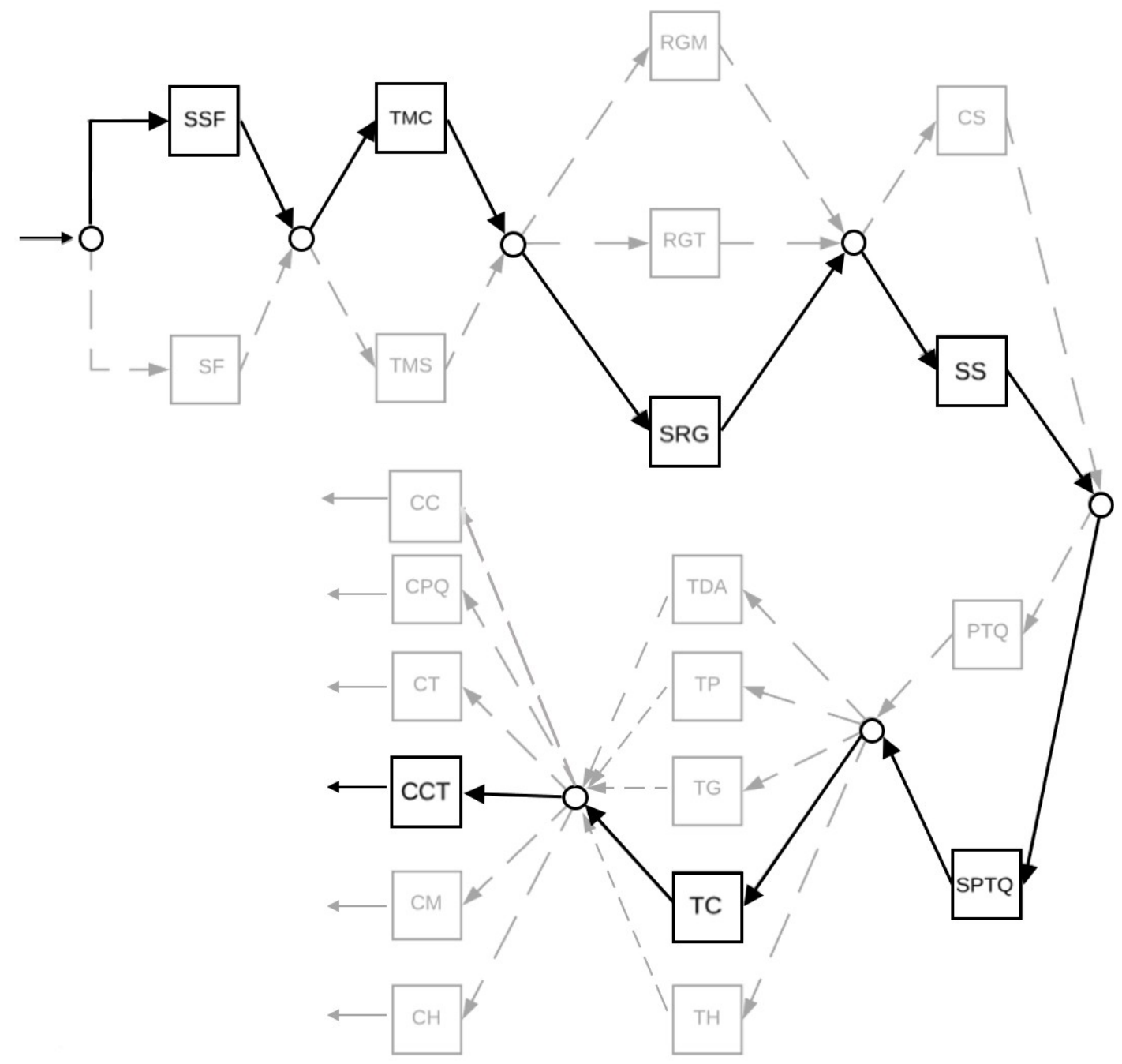

Figura 4 Fluxograma mais promissor pelo método heurístico representado na superestrutura

\section{CONCLUSÃO}

No presente estudo o cenário do RSU no Rio de Janeiro foi caracterizado. Foi evidenciado a grande quantidade de RSUs gerados anualmente, e problemas na gestão dos 
resíduos, como a baixa abrangência da coleta seletiva e praticamente inexistência de reaproveitamento energético. Por meio da revisão bibliográfica foram identificadas as principais tecnologias usadas mundialmente no reaproveitamento energético do RSU com seus principais aspectos e limitações. As cinco tecnologias identificadas foram hierarquizadas pelo potencial de produção energética por tonelada de RSU tratado.

A partir das tecnologias identificadas o processo de produção de energia a partir de RSU foi dividido em subsistemas, o que possibilitou a síntese de fluxogramas alternativos, representados pela superestrutura. Nesse contexto foram estabelecidas regras heurísticas a fim de se identificar o fluxograma mais promissor de acordo com o método heurístico.

O fluxograma encontrado - SSF, TMC, SRG, SS, SPTQ, TC, CCT - apresentou um potencial de geração energética para o estado do Rio de Janeiro de 14.256.095 MWh. Para esse potencial, considera-se que $100 \%$ dos RSUs do estado são coletados e encaminhados para o tratamento por combustão. Esse potencial corresponde à $94 \%$ da demanda energética da cidade do Rio de Janeiro.

\section{REFERÊNCIAS BIBLIOGRÁFICAS}

[1] ARENA, Umberto. Process and technological aspects of municipal solid waste gasification. A review. Waste management, [S.I], v. 32, n. 4, p. 625-639, abr. 2012.

[2] BRASIL. Lei no 12.305, de 2 de agosto de 2010. Institui a Política Nacional de Resíduos Sólidos. Diário Oficial da República Federativa do Brasil, Brasília, DF, 2 ago. 2010. Disponível em: <https://www.planalto.gov.br/ccivil_03/_ato20072010/2010/lei/l12305.htm>. Acesso em 23 ago 2018.

[3] EPA (ENVIRONMENTAL PROTECTION AGENCY). U. S. Anaerobic Digestion Facilities Processing Food Waste in the United States in 2015: Survey Results. 2018. Disponível em: <https://www.epa.gov/> Acesso em 14 jan 2019

[4] EUROPEAN COMMISSION. Integrated Pollution Prevention and Control: Reference Document on the Best Available Techniques for Waste Incineration., Bruxelas, European Commission, 2006a.

[5] EUROPEAN COMMISSION. Integrated Pollution Prevention and Control Reference: Document on the Best Available Techniques for Waste Treatments Industries. Bruxelas: European Commission, 2006b.

[6] HOGG, D. et al. Economic analysis of options for managing biodegradable municipal waste-Final report. EC report, [S.1], 2002.

[7] HOORNWEG, Daniel; Bhada-Tata, Perinaz. What a Waste: A Global Review of Solid Waste Management. Washington, DC: World Bank, 2012. (Urban development series; knowledge papers no. 15)

[8] ISWA (INTERNATIONAL SOLID WASTE ASSOCIATION). Waste-to-Energy: State-of-the-Art-Report. 6. ed., [S.l], 2012.

[9] KALOGIROU, Efstratios N. Waste-to-Energy technologies and global applications. Boca Raton: CRC Press, 2018. 244p.

[10] KHAN, M. Rashid. Clean energy from waste and coal. Washington, DC: American Chemical Society, 1992. (ACS Symposium series) 
[11] PEDROZA, Marcelo Mendes et al. Aproveitamento energético de resíduos sólidos urbanos em processo de pirólise. Revista Brasileira de Energias Renováveis, [S.l.], v. 6, n. 2, 2017.

[12] ROGOFF, Marc J.; SCREVE, Francois. Waste-to-energy: technologies and project implementation. 2. ed. Waltham: William Andrew, 2011.

[13] SEA (SECRETARIA DE ESTADO DO AMBIENTE). Plano Estadual de Resíduos Sólidos do Rio de Janeiro (PERS): Relatório Síntese, Rio de Janeiro, 2013. Disponível em: <http://www.rj.gov.br/web/sea/exibeconteudo?article-id=1941406> Acesso em 02 set 2018. 